\title{
Evaluation of the Early Stage of Carotid Atherosclerosis Using the Vascular Response to Nitroglycerin and High-Resolution Ultrasonography
}

\author{
Cha-Po Lai, MD; Samon Koyanagi, MD**; Cheng-Kuang Shaw, DDS*; Akira Takeshita, MD**
}

\begin{abstract}
The purpose of this study was to determine whether the early stages of arterial atherosclerosis could be evaluated by the vascular response to nitroglycerin (NTG). The vascular elasticity of the common carotid artery (CCA) was evaluated at sites without atheromatous plaque using high-resolution ultrasonography before and after sublingular NTG administration. The vascular elasticity was expressed by vascular strain (VS) and pressurestrain elastic modulus (Ep). The study was performed in 9 normal young males (group N) and 52 patients who were suspected to have coronary artery disease (CAD). In group N, VS of the CCA increased from $9 \pm 2$ to $13 \pm 4 \%$, Ep decreased from $80 \pm 18$ to $42 \pm 14 \mathrm{kPa}(\mathrm{p}<0.01)$ and systolic blood pressure $(\mathrm{SBP})$ decreased from $116 \pm 8$ to $109 \pm 8 \mathrm{mmHg}(\mathrm{p}<0.05)$ after NTG administration. Two different responses were observed in the patients. One group of patients (group A, $n=27$ ) showed similar responses to NTG as those of group N; that is, VS increased from $8 \pm 4$ to $14 \pm 8 \%$, Ep decreased from $115 \pm 64$ to $57 \pm 31 \mathrm{kPa}(\mathrm{p}<0.01)$ and SBP decreased from $121 \pm 15$ to $110 \pm 16 \mathrm{mmHg}(\mathrm{p}<0.05$ ). In the other group (group B, $\mathrm{n}=25$ ), in spite of SBP decreasing from $124 \pm 21$ to $111 \pm 21 \mathrm{mmHg}(\mathrm{p}<0.05)$, VS decreased from $11 \pm 4$ to $7 \pm 3 \%$ and Ep increased from $76 \pm 29$ to $113 \pm 53 \mathrm{kPa}$ $(\mathrm{p}<0.01)$. The prevalence and severity of CAD were lower in group A than in group B (33 vs $80 \%, 11 \mathrm{vs} 60 \%$, $\mathrm{p}<0.01$, respectively). The prevalence of atheromatous plaque of the CCA was also lower in group A than in group B (4 vs $22 \%, \mathrm{p}<0.01)$. These results revealed that the prevalence of carotid atheromatous plaque was highly correlated with that of CAD $(\mathrm{p}=0.001)$ and suggested that the vascular elasticity response of the CCA to NTG is highly associated with atherosclerotic changes of the artery and may be a useful method for detecting the early stages of atherosclerosis. (Jpn Circ J 1998; 62: 494-498)
\end{abstract}

Key Words: Atherosclerosis; Nitroglycerin; Vascular elasticity

\section{A} therosclerosis is a series of histopathological changes that includes intimal thickening, degenerative compositional changes of the medial layer (sclerotic change) and atheroma-formation (atherotic change)! The recent development of high-resolution Bmode ultrasonography has made it possible to detect and evaluate the atheromatous changes of the arteries, particulary the carotid artery $?^{2-5}$ However, despite these studies, methods that evaluate the sclerotic change of an artery, an early stage of atherosclerosis, have not been established. Vascular sclerotic changes increase vascular stiffness, and may disturb the vascular responses to a vascular relaxant. Although decreased elasticity of the arterial wall in the atherosclerotic carotid artery has been reported ${ }^{6-9}$ the carotid artery response to vascular relaxant has not been reported yet.

Nitroglycerin (NTG), a relaxant of vascular smooth muscle, may change arterial elasticity 10,11 If the change in elasticity of the atherosclerotic artery in response to NTG is different from that of the normal artery, evaluation of this

(Received August 14, 1997; revised manuscript received March 2, 1998; accepted March 5, 1998)

Division of Cardiology, Department of Medicine, Buddhist Tzu-Chi General Hospital, Taiwan, *Department of Public Health, Tzu-Chi College of Medicine, Taiwan and**Research Institute of Angiocardiology and Cardiovascular Clinic, Faculty of Medicine, Kyushu University, Fukuoka, Japan

Mailing address: Cha-Po Lai, MD, Buddhist Tzu Chi General Hospital, 707 Chung Yan Road, Sec.3, Hualien, Taiwan response may be valuable in assessing atherosclerotic changes. The purpose of the present study was to determine whether the early atherosclerotic changes of the common carotid artery (CCA) could be evaluated by the response of vascular elasticity to NTG, where there was no ultrasonic atheromatous plaque. The vascular elasticity was expressed by vascular strain (VS) and pressure-strain elastic modulus (Ep). Moreover, the extent of atherosclerosis of the CCA has been shown to be strongly correlated with the extent of coronary artery disease (CAD) $!^{2-16}$ Therefore, we also examined whether the decreased elasticity response of the CCA to NTG was associated with CAD.

\section{Methods}

\section{Study Subjects}

The study was performed in 52 patients who were admitted for the evaluation of CAD ( 35 men and 17 women, aged from 45 to 74 years old ), and 9 normal male volunteers (aged from 23 to 27 years old) without risk factors for atherosclerosis such as hyperlipidemia, hypertension, glucose intolerance and smoking. Patients with frequent arrhythmias, congestive heart failure, congenital and acquired heart disease were excluded, because the hemodynamic abnormality of these diseases may affect the diameter change of the CCA. Patients with recent cerebrovascular attacks and a blood pressure difference between the bilateral brachial artery of greater than $10 \mathrm{mmHg}$ were also excluded. Informed consent was obtained from every 


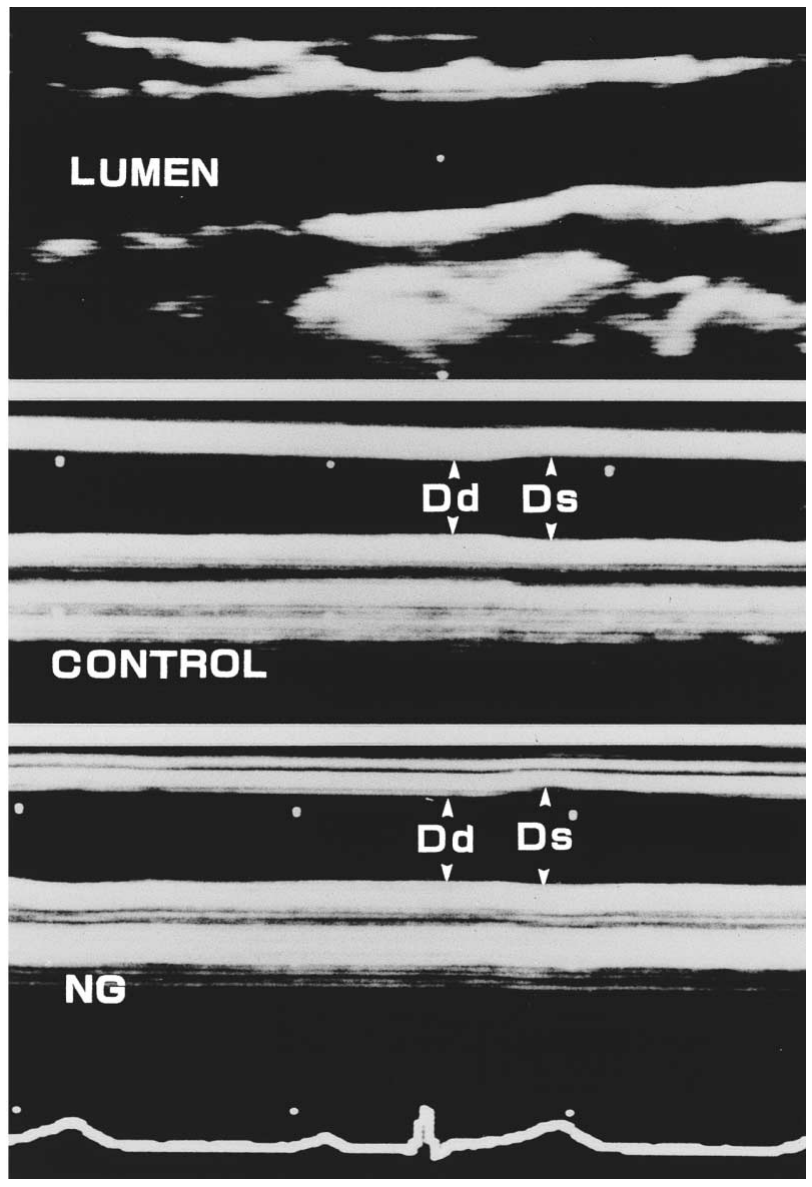

Fig 1. The cursor of the M-mode was placed $3 \mathrm{~cm}$ proximal to the carotid bifurcation where there was no atheromatous plaque (upper), and the maximum diameter of the artery were recorded before (CONTROL) and after (NG) nitroglycerin sublingular administration. Dd, end diastolic diameter; Ds, end systolic diameter.

patient.

All the 52 patients were suspected to have CAD and underwent coronary arteriography to assess the extent of it. A significant stenosis was defined as a greater than 50\% decrease in the internal diameter. There were 29 patients with significant coronary stenosis.

\section{Examination of the Carotid Arteries}

None of the patients stopped their regular medications, such as nitrates, calcium antagonists and/or beta blockers, for fear of ischemic and withdrawal hypertensive attacks. The protocol began in the morning, $2 \mathrm{~h}$ after medications were taken.

The ultrasonographic studies were performed using a Bmode and M-mode ultrasonographic instrument (SSD7255, Aloka, Tokyo, Japan) with a $10 \mathrm{MHZ}$ frequency of the mechanical sector scanner. Subjects were placed in the supine position with their head tilted slightly to the right, and the left CCAwas investigated. We scanned the CCA from the carotid bifurcation to the proximal portion as far as possible in 3 longitudinal sections (anterior oblique, lateral and posterior oblique) and in transverse section, and the presence of atheromatous plaque was evaluated. Atheromatous plaque was thought to be present if the
Table 1 Hemodynamic and Vascular Elasticity Responses to NTG in Normal Young Males

\begin{tabular}{lcrccccc}
\hline \hline No. & $\begin{array}{c}\text { Age } \\
\text { (years) }\end{array}$ & & $\begin{array}{c}S B P / D B P \\
(\mathrm{mmHg})\end{array}$ & $\begin{array}{c}P P \\
(\mathrm{mmHg})\end{array}$ & $\begin{array}{c}D \\
(\mathrm{~mm})\end{array}$ & $\begin{array}{c}V S \\
(\%)\end{array}$ & $\begin{array}{c}E p \\
(\mathrm{kPa})\end{array}$ \\
\hline 1. & 25 & $C$ & $122 / 58$ & 64 & 6.8 & 9 & 97 \\
& & $N T G$ & $116 / 79$ & 37 & 6.7 & 12 & 42 \\
2. & 25 & $C$ & $108 / 70$ & 38 & 6.6 & 8 & 68 \\
& & $N T G$ & $101 / 64$ & 37 & 6.3 & 8 & 64 \\
3. & 27 & $C$ & $118 / 78$ & 40 & 5.3 & 9 & 60 \\
& & $N T G$ & $110 / 78$ & 32 & 5.3 & 14 & 74 \\
4. & 23 & $C$ & $132 / 71$ & 61 & 6.1 & 10 & 84 \\
& & $N T G$ & $125 / 81$ & 44 & 6.3 & 11 & 55 \\
5. & 26 & $C$ & $114 / 69$ & 45 & 6.6 & 6 & 103 \\
& & $N T G$ & $106 / 73$ & 33 & 6.6 & 8 & 56 \\
6. & 26 & $C$ & $110 / 58$ & 52 & 5.4 & 7 & 101 \\
& & $N T G$ & $104 / 67$ & 37 & 6.2 & 14 & 36 \\
7. & 24 & $C$ & $115 / 66$ & 49 & 6.2 & 13 & 52 \\
& & $N T G$ & $105 / 72$ & 33 & 6.0 & 18 & 25 \\
8. & 25 & $C$ & $108 / 56$ & 52 & 5.4 & 9 & 79 \\
& & $N T G$ & $104 / 67$ & 37 & 5.9 & 18 & 29 \\
9. & 24 & $C$ & $118 / 63$ & 55 & 6.6 & 10 & 75 \\
& & $N T G$ & $112 / 72$ & 40 & 6.6 & 15 & 37 \\
\hline
\end{tabular}

$C$, before nitroglycerin; NTG, after NTG administration; SBP, systolic blood pressure; DBP, diastolic blood pressure; PP, pulse pressure; $D$, internal diameter of common carotid artery; VS, vascular strain; Ep, pressure-strain elastic modulus.

echogenic lesion was detected in at least one of the longitudinal sections and in the transverse section. In order to measure the diameter of the CCA, the cursor of the Mmode was placed approximately $3 \mathrm{~cm}$ proximal to the carotid bifurcation, where there was no ultrasonic evidence of atheromatous plaque, and the maximum diameter of the artery was recorded. The pulse rate (PR) and blood pressure (BP) of the brachial artery were noninvasively recorded every minute using a monitoring system (STBP680 Nippon Colin Inc., Tokyo, Japan).

\section{Administration of Nitroglycerin}

NTG was given sublingually $(0.3 \mathrm{mg})$ and $\mathrm{BP}$ were recorded every minute thereafter. When the maximal $\mathrm{BP}$ response was obtained, the diameter of the CCA was recorded.

\section{Data Analysis}

The internal diameter (D) of the CCA was measured in 3 consecutive cardiac cycles of M-mode echograms. Optimal gain setting of the sonograph was obtained to measure the internal diameter from the leading edge of the intimal echo of the near arterial wall to the leading edge of the intimal echo of the far wall. The smallest and the largest internal diameters were measured at end-diastole and peak systole, respectively, as shown in Fig 1. Two independent observers measured twice the internal diameter of all ultrasonograms of the CCA, with a 1-week interval. The interobserver reliability expressed as the correlation between these 2 observers was satisfactory $(\mathrm{r}=0.85)$. Paired $\mathrm{t}$-test was used to compare the intraobserver differences in the measurement of carotid D. The results showed that the value measured by these 2 observers was consistant $(\mathrm{p}>0.05)$. Intraobserver differences for the measurement were compared using paired t-test. There was no significant difference between these 2 observers ( $p>0.05)$. The VS and Ep of the CCA were calculated according to the following formulae: 
Table 2 Hemodynamics, Arterial Diameter, Vascular Elasticity of Group N and Patient Groups A and B Before and After NTG

\begin{tabular}{cccccccc}
\hline \hline Group & & $\begin{array}{c}\text { SBP/DBP } \\
(\mathrm{mmHg})\end{array}$ & $\begin{array}{c}P P \\
(\mathrm{mmHg})\end{array}$ & $\begin{array}{c}P R \\
(\mathrm{bpm})\end{array}$ & $\begin{array}{c}D \\
(\mathrm{~mm})\end{array}$ & $\begin{array}{c}V S \\
(\%)\end{array}$ & $\begin{array}{c}E p \\
(\mathrm{kPa})\end{array}$ \\
\hline \multirow{2}{*}{$N$} & $C$ & $116 \pm 8 / 65 \pm 7$ & $51 \pm 9$ & $68 \pm 11$ & $6.1 \pm 0.6$ & $9 \pm 2$ & $80 \pm 18$ \\
& $N T G$ & $109 \pm 8 * / 73 \pm 6$ & $37 \pm 4^{\dagger}$ & $70 \pm 12$ & $6.2 \pm 0.4$ & $13 \pm 4^{\dagger}$ & $42 \pm 14^{\dagger}$ \\
$A$ & $C$ & $121 \pm 15 / 69 \pm 11$ & $52 \pm 12$ & $69 \pm 12$ & $6.2 \pm 1.0$ & $8 \pm 4$ & $115 \pm 64$ \\
& $N T G$ & $110 \pm 16^{*} / 70 \pm 11$ & $40 \pm 12^{\dagger}$ & $71 \pm 11$ & $6.1 \pm 0.8$ & $14 \pm 8^{\dagger}$ & $57 \pm 31^{\dagger}$ \\
$B$ & $C$ & $124 \pm 21 / 66 \pm 14$ & $58 \pm 15$ & $67 \pm 11$ & $6.1 \pm 1.3$ & $11 \pm 4$ & $76 \pm 29$ \\
& $N T G$ & $111 \pm 21^{*} / 68 \pm 10$ & $43 \pm 11^{\dagger}$ & $69 \pm 12$ & $6.2 \pm 1.4$ & $7 \pm 3^{\dagger}$ & $113 \pm 53^{\dagger}$ \\
\hline
\end{tabular}

Mean $\pm S D$

$C$, before nitroglycerin; NTG, after nitroglycerin administration; SBP, systolic blood pressure; DBP, diastolic blood pressure; PP, pulse pressure; $P R$, pulse rate; $D$, end diastolic internal diameter of common carotid artery; VS, vascular strain; Ep, pressurestrain elastic modulus; ${ }^{*} p<0.05$ v data before NTG in the same group; ${ }^{\dagger} p 0.01$ vs data before NTG in the same group.

Table 3 The Prevalence of Atheromatous Plaque of the Common Carotid Artery and the Extent of Coronary Artery Disease in Groups A and B

\begin{tabular}{lcc}
\hline \hline & $\begin{array}{c}\text { Group A } \\
(n=27)\end{array}$ & $\begin{array}{c}\text { Group B } \\
(n=25)\end{array}$ \\
\hline Averaged age (years) & $57 \pm 10$ & $62 \pm 10^{*}$ \\
Atheromatous plaque (persons, \%) & $6(22 \%)$ & $21(84 \%)^{*}$ \\
Coronary artery disease (persons, \%) & $9(33 \%)$ & $20(80 \%)^{*}$ \\
$\quad$ single vessel disease & $8(30 \%)$ & $8(32 \%)$ \\
multi vessel disease & $1(4 \%)$ & $12(48 \%)^{*}$ \\
\hline
\end{tabular}

$* p<0.01$ vs group $A$.

VS $(\%)=\left(\right.$ systolic D - diastolic D) $\mathrm{mm} /$ diastolic $\mathrm{D} \mathrm{mm} \mathrm{m}^{17}$ $\mathrm{Ep}(\mathrm{kPa})=($ systolic $\mathrm{BP}-$ diastolic BP $) \mathrm{mmHg} / 7.6 \mathrm{mmHg}$ per $\mathrm{VS}^{7}$

The data were expressed as mean \pm SD (standard deviation). Paired t-test was used to compare hemodynamics, end diastolic D, VS and Ep before and after NTG administration. Chi square test was used to analyze the prevalence of $\mathrm{CAD}$ and carotid atheromatous plaque between groups. A probability of less than 0.05 was considered significant.

\section{Results}

Table 1 reveals the clinical data of the 9 normal young males (group N) before and after NTG administration. BP, pulse pressure (PP) and Ep decreased, while VS increased after NTG in all subjects but one, in whom only strain was unchanged (case No 2). Hemodynamics, diastolic D, VS and $\mathrm{Ep}$ were compared in group $\mathrm{N}$ and the 52 patients with suspected CAD before and after NTG administration (Table 2). In group N, the SBP, PP and Ep decreased significantly $(\mathrm{p}<0.05, \mathrm{p}<0.01, \mathrm{p}<0.01$, respectively), and VS increased significantly $(\mathrm{p}<0.01)$ after NTG. However, no significant change in PR and diastolic D were found after NTG administration.

In the patients who underwent coronary arteriography for the evaluation of CAD $(n=52), B P$ and PP decreased after NTG administration, as in group N. However, VS and Ep changed in various ways. We divided the patients into 2 groups by the different responses to NTG: (i) there were 27 patients (18 men and 9 women; mean age, $57 \pm 10$ years) who showed a decrease in Ep and an increase in VS, as in group N (group A), and (ii) 25 patients (17 men and 8 women; mean age: $62 \pm 10$ years) who showed an increase in Ep and a decrease in VS (group B). There were also no significant changes in diastolic D before and after NTG administration among the patients in these groups. As in
Table 4 The Prevalence Atheromatous Plaque and the Extent of Coronary Artery Disease in Group A and B After Age Matched Comparison

\begin{tabular}{lcc}
\hline \hline & $\begin{array}{c}\text { Group A } \\
(n=14)\end{array}$ & $\begin{array}{c}\text { Group B } \\
(n=22)\end{array}$ \\
\hline Atheromatous plaque (persons, \%) & $4(29 \%)$ & $17(77 \%)^{*}$ \\
Coronary artery disaese (persons, \%) & $3(21 \%)$ & $17(77 \%)^{*}$ \\
$\quad$ single vessel disease & $3(21 \%)$ & $5(23 \%)$ \\
multi vessel disease & $0(0 \%)$ & $12(55 \%)^{*}$ \\
\hline
\end{tabular}

$*_{p}<0.01$ vs group A.

group N, NTG decreased SBP and PP significantly in both group A and B. The changes in SBP and PP were not significantly different when compared between any 2 of the 3 groups. In group A, Ep decreased from $115 \pm 64$ to $57 \pm 31$ $\mathrm{kPa}(\mathrm{p}<0.01)$ and VS increased from $8 \pm 4$ to $14 \pm 8 \%$ $(\mathrm{p}<0.01)$. In group B, Ep incresed from $76 \pm 29$ to $113 \pm 53$ $\mathrm{kPa}(\mathrm{p}<0.01)$, and VS decreased from $11 \pm 4$ to $7 \pm 3 \%$ significantly $(\mathrm{p}<0.01)$. However, there were no statistical differences in the basal values of Ep and VS when compared between any 2 of the 3 groups.

The extent of atheromatous plaque in the carotid artery and CAD were compared between groups A and B (Table 3 ). Atheromatous plaque of the carotid artery was not observed in any group $\mathrm{N}$ subject. The prevalence of atheromatous plaque was substantially lower in group A than in group B ( 22 vs $84 \%, p<0.01)$. In group A, there were 9 patients $(33 \%)$ with CAD and only 1 patient had multivessel disease $(4 \%)$. In group B, however, there were 20 patients $(80 \%)$ with CAD and 12 patients $(48 \%)$ had multivessel disease. The prevalence and severity of CAD were statistically significant when compared between group A and $B(p<0.01)$. Because the average age of patients in group B was higher than in group A, an age-matched comparison was made and is shown in Table 4. Even after correction for age, the prevalence of carotid atheromatous plaque and $\mathrm{CAD}$ were significantly higher in group $\mathrm{B}$ than in group A ( 77 vs $29 \%$; 77 vs $21 \%$, respectively, $\mathrm{p}<0.01$ ).

The prevalence of carotid atheromatous plaque was highly correlated with CAD ( $\mathrm{p}=0.001)$.

\section{Discussion}

The present study has shown that in patients suspected of having CAD there were 2 different responses of the CCA to NTG, when the vascular elasticity was evaluated at a site without ultrasonic evidence of atheromatous plaque. One 
group of patients (group A) showed a decrease in Ep and an increase in VS after NTG administration, similar to the response in group $\mathrm{N}$, whereas the other group (group B) showed an increase in Ep and a decrease in VS, although SBP and PP decreased after NTG administration in both groups. It appeared that group B had more extensive and severe atherosclerotic changes of the carotid and coronary arteries than group A. These results indicate that the Ep and VS responses of the CCA to NTG were strongly associated with atherosclerotic changes.

The reliability of this study was examined. High resolution ultrasonography with a $10 \mathrm{MHZ}$ mechanical scanner was used to evaluate the internal diameter change of the carotid artery and this system provides a longitudinal resolution of $0.2 \mathrm{~mm}$. Moreover, the measured value of 2 independent observers was consistent $(p>0.05)$. However, instead of intracarotid pressure, the brachial artery was used to measure the BP. The limitation of the present study was that the relationship between the brachial artery BP and intracarotid pressure was not evaluated, because of the risk of directly measuring intracarotid pressure.

The extent of atheroscleosis of the CCA has been evaluated by morphological changes, including plaque formation and intima-media thickness (IMT)4 Atherosclerotic changes may alter the physiological properties of the artery wall even where morphological changes are minimal? Moreover, Barth et al have evaluated the pulsatile change of the carotid artery diameter and shown that the pressurestrain modulus was greater at the atherosclerotic lesion than at the nonatherosclerotic sites? Thus, it is possible that the physiological properties of the artery were modified by vasoactive drugs, with which early atherosclerotic changes could be clarified, but this has not been reported previously. We evaluated the changes in VS and Ep of the CCA by the effects of NTG, and have shown that even at the nonatheromatous sites the responses were significantly different between patients with and without extensive atherosclerosis.

VS is a percent change of vascular diameter during the systolic phase, which is determined by BP, especially systemic BP (SBP), and vascular wall properities 18-20 $^{18}$ Vascular wall is composed of smooth muscle and connective tissue components ${ }^{18,19}$ In the elastic type of artery, vascular elasticity is mainly modulated by the smooth muscle component 18,21 We also measured the Ep to estimate the arterial elasticity, because Ep is determined with data that normalize for PP and vascular diameter differences between subjects, and is commonly used in population surveys? A previous experiment showed that NTG decreases the pulse-wave velocity of the aorta, suggestive of a decrease in arterial stiffness ${ }^{10}$ As the vascular elasticity is determinated by BP and vascular properties, ${ }^{18-20}$ the decreased pulse-wave velocity might be due to a NTGinduced decrease in BP, rather than by vascular smooth muscle relaxation. However, $0.3 \mathrm{mg}$ of NTG was administrated sublingually in our study and, although we did not examine the plasma concentration of NTG during the study, the decrease in SBP and PP was similar between groups A and B. Therefore, the different responses of VS and Ep to NTG between these 2 groups can not be explained by a decrease in BP induced by NTG.

In the present study, although NTG decreased SBP, VS increased and Ep decreased in groups $\mathrm{N}$ and $\mathrm{A}$. These results suggest that NTG decreases the elasticity of CCA in those patients who have less atherosclerotic changes through the direct effect of NTG on vascular smooth muscle, which results in an increase in vascular elasticity. In group B, VS decreased and Ep increased after NTG administration. Although the reason for this is unclear, it indicates that the relaxing effect of NTG may have been limited in spite of the fact that atheromatous plaque was not detected at the measured sites of the CCA. It has been reported that the IMT measurement was a useful method for detecting the early stage of atherosclerosis.,22 Increased IMT may be an important factor in disturbing the effect of NTG, although IMT was not evaluated in the present study.

Finally, our results identified that there was a high correlation between CAD and atheromatous plaque, ${ }^{13,16}$ and revealed that it is possible to evaluate the vascular atherosclerotic change at an early stage using this noninvasive and safe method. Moreover, this method does not use expensive instruments. From point of view of preventing the progression of atherosclerosis, it is an easy and useful method for evaluating early stage vascular atherosclerosis.

Vascular atherosclerosis regresses after hyperlipidemia is adjusted22,23 Further studies are needed to determine whether the vascular elasticity response to NTG will change if the risk factors for atherosclerosis are controlled.

\section{References}

1. Blankenhorn DH, Kramsch DM: Reversal of atherosis and sclerosis The two components of atherosclerosis. Circulation 1989; 79: 1-7

2. Crouse JR, Harpold GH, Kahl FR, Toole JF, Mckinney WM: Evaluation of a scoring system for extracranial carotid atherosclerosis extent with B-mode ultrasound. Stroke 1986; 17: 270-275

3. Salonen R, Salonen JT: Progression of carotid atherosclerosis and its determinants: a population-based ultrasonography study. Atherosclerosis 1990; 81: 33-40

4. Salonen JT, Salonen R: Ultrasound B-mode imaging in observational studies of atherosclerotic progression. Circulation 1993; 87(Suppl II): $56-65$

5. Zanette E, Bozzao L, Buttinelli C, Mariottini A, Pappata S, Lenzi GL: High resolution real-time B-mode echography in the diagnosis of extracranial carotid lesions: Comparison with traditional angiography. Acta Neurochir 1987; 84: 43-47

6. Spagnoli LG, Bonanno E, Mauriello A, Zanette E, Faraglia V Palmieri G, Fiorani P: Histopathological characterization of carotid plaque echotomography. Int Angiol 1988; 7: 75-80

7. Riley WA, Freedman DS, Higgs NA, Barnes RW, Zinkgraf SA Berenson GS: Decreased arterial elasticity associated with cardiovascular disease risk factors in the young: Bogalusa Heart Study. Arteriosclerosis 1986; 6: $378-386$

8. Barth JD, Blankenhorn DH, Wickham E, Lai JY, Chin HP, Selzer RH: Quantitative ultrasound pulsation study in human carotid artery disease. Arteriosclerosis 1988; 8: 778-781

9. Hirai T, Sasayama S, Kawasaki T, Yagi S: Stiffness of systemic arteries in patients with myocardial infarction. Circulation 1989; 80: $78-86$

10. Latson TW, Hunter WC, Katoh N, Sagawa K: Effect of nitroglycerin on aortic impedance, diameter, and pulse-wave velocity. Circulation Res 1988; 62: $884-890$

11. Smulyan H, Mookherjce S, Warner RA: The effect of nitroglycerin on forearm arterial distensibility. Circulation 1986; 73: 1264-1269

12. Crouse JR, Thompson CJ: An evaluation of methods for imaging and quantifying coronary and carotid lumen stenosis and ahterosclerosis. Circulation 1993; 87(Suppl II): 17-33

13. Craven TE, Ryu IE, Espeland MA, Kahl FR, McKinney WM, Toole JF et al: Evaluation of the associations between carotid artery atherosclerosis and coronary artery stenosis: A case-control study. Circulation 1990; 82: 1230-1242

14. Pasquale GD, Andreoli A, Pinelli G, Grazi P, Manini G, Tognetti F et al: Cerebal ischemia and asymptomatic coronary artery disease: A prospective study of 83 patients. Stroke 1986; 17: 1098-1101

15. Salonen JT, Salonen R: Ultrasonograghically assessed carotid morphology and the risk of coronary heart disease. Arterioscler Thromb 1991; 11: $1245-1249$

16. Wofford JL, Kahl FR: Howard GR, McKinney WM, Tool JF, Crouse JR 3rd. Relation of extent of extracranial carotid artery atherosclero- 
sis as measured by B-mode ultrasound to the extent of coronary atherosclerosis. Arterioscler Thromb 1991; 11: 1786-1794

17. Aberg AK, Axelsson J: Some mechanical aspects of an intestinal smooth muscle. Acta Physiol Scand 1965; 64: 15-27

18. Dobrin PB, Rovick AA: Influence of vascular smooth muscle on contractile mechanics and elasticity of arteries. Am J Physiol 1969; 217: $1644-1651$

19. Hayashi K, Sato M, Handa H, Moritake K: Biomechanical study of the constitutive laws of vascular walls. Exp Mechanics 1974; 14: $440-444$

20. Cox RT: Comparison of arterial wall mechaics in normotensive and spontaneously hypertensive rats. Am J Physiol 1979; H159-H167

21. Cox RT: Alterations in active and passive mechanics of rat carotid artery with experimental hypertension. Am J Physiol 1979; H597H605

22. Byington RP, Furberg CD, Crouse JR, Bond MG: Pravastatin, lipids, and atherosclerosis in the carotid arteries (PLAC II). Am J Cardiol 1995; 76: 54C-59C

23. Farrar DJ, Green HD, Wagner WD, Bond MG: Reduction in pulse wave velocity and improvement of aortic distensibility accompanying regression of atherosclerosis in the Rhesus monkey. Circ Res 1980; 45: 425-432 